\title{
Primal Separation for 0/1 Polytopes
}

\author{
Friedrich Eisenbrand* \\ Max-Planck-Institut für Informatik \\ Stuhlsatzenhausweg 85 \\ 66123 Saarbrücken \\ Germany \\ eisen@mpi-sb.mpg.de
}

\author{
Giovanni Rinaldi, Paolo Ventura \\ Istituto di Analisi dei Sistemi ed Informatica \\ Viale Manzoni, 30 \\ 00185 Roma \\ Italy \\ [rinaldi, ventura] @iasi.rm.cnr.it
}

June 25, 2002

\begin{abstract}
The $0 / 1$ primal separation problem is: Given an extreme point $\bar{x}$ of a $0 / 1$ polytope $P$ and some point $x^{*}$, find an inequality which is tight at $\bar{x}$, violated by $x^{*}$ and valid for $P$ or assert that no such inequality exists. It is known that this separation variant can be reduced to the standard separation problem for $P$.

We show that $0 / 1$ optimization and $0 / 1$ primal separation are polynomial time equivalent. This implies that the problems $0 / 1$ optimization, $0 / 1$ standard separation, $0 / 1$ augmentation, and $0 / 1$ primal separation are polynomial time equivalent.

Then we provide polynomial time primal separation procedures for matching, stable set, maximum cut, and maximum bipartite graph problems, giving evidence that these algorithms are conceptually simpler and easier to implement than their corresponding counterparts for standard separation. In particular, for perfect matching we present an algorithm for primal separation that rests only on simple max-flow computations. In contrast, the known standard separation method relies on an explicit minimum odd cut algorithm. Consequently, we obtain a very simple proof that a maximum weight perfect matching of a graph can be computed in polynomial time.
\end{abstract}

\section{Introduction}

The polynomial time equivalence of separation and optimization (Grötschel, Lovász \& Schrijver 1981, Padberg \& Rao 1981, Karp \& Papadimitriou 1980) provides a powerful tool to prove that certain combinatorial optimization problems can be solved in polynomial time, such as finding shortest paths, submodular function minimization or computing maximal stable sets in perfect graphs among many others.

Perhaps one of the most stunning applications of this equivalence is the proof of Padberg \& Rao (1982) that maximum weight matchings can be computed in polynomial time. Padberg and Rao used the inequality description of the convex hull of characteristic vectors of perfect matchings (Edmonds 1965a) and the equivalence of separation and optimization to reduce the maximum weighted perfect matching problem to the problem of finding a minimal odd cut of a graph. Their separation algorithm is much simpler than Edmonds's (1965b) blossom shrink algorithm, which solves the maximum weighted matching problem directly.

\footnotetext{
${ }^{*}$ Research developed while the author was on leave at the Istituto di Analisi dei Sistemi ed Informatica, supported by the project TMR-DONET nr. ERB FMRX-CT98-0202 of the European Union.
} 


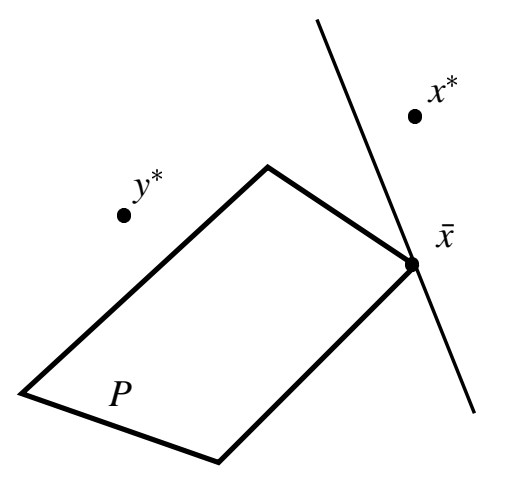

Figure 1: A primal cutting plane of $\bar{x}$ separating $x^{*}$ from $P$. Notice that $y^{*}$ cannot be separated from $P$ by a primal cutting plane of $\bar{x}$.

In this paper we consider the following variant of the separation problem, called primal separation. Here, given an extreme point $\bar{x}$ of an integral polyhedron $P$ and some point $x^{*}$, one has to compute an inequality $a^{T} x \leq \beta$, which is valid for $P$, tight at $\bar{x}$ (i.e., $a^{T} \bar{x}=\beta$ ) and not valid for $x^{*}$ or assert that no such inequality exists. An inequality $a^{T} x \leq \beta$ meeting these conditions is called a primal cutting plane. If one does not require tightness $a^{T} \bar{x}=\beta$ of the valid inequality, we speak about the standard separation problem and a standard cutting plane. Notice that if $x^{*}$ is not in $P$, then there is always a standard cutting plane separating $x^{*}$ from $P$ whereas this does not hold in the primal case, see Figure 1.

Primal separation appeared first in the context of an integer programming algorithm based on simplex-like pivoting (Young 1968). By linear programming duality, primal separation is the method of choice to prove optimality of an integer point $\bar{x}$. Padberg \& Hong (1980) investigated the potential of this observation to prove optimality of traveling salesman tours. In contrast to Young (1968), they used inequalities defining facets of the traveling salesman polytope as primal cutting planes.

Padberg and Hong's approach for the traveling salesman problem, like many algorithms for polynomially solvable combinatorial problems, is of primal flavor. It starts from an integral feasible solution and then either proves that such a solution is optimal, or provides a current linear programming relaxation to the problem with enough inequalities, generated by separation, to perform a pivot that produces a better integral feasible solution. The use of separation in this "primal" environment, where all the generated inequalities must satisfy the current solution at equality, motivates the term "primal separation" that has been used in the above definition and is now commonly used terminology.

If we exclude very few exceptions (see, e.g., De Simone \& Rinaldi (1994)), the primal approach of Padberg and Hong had a very limited follow-up. Indeed, all the polyhedral-based cutting plane algorithms for NP-hard integer programming or combinatorial optimization problems are based on a dual approach: a sequence of infeasible solutions is generated; each of them is the optimum of the current linear programming relaxation of the problem. Each solution of the sequence is in turn separated from the integral feasible solutions by an inequality produced by standard separation. Optimality is reached as soon as the optimum of the current relaxation is integral.

Recently, the primal approach and primal cutting planes are reconsidered again, also due to their close connection to augmentation algorithms, see (Firla, Spille \& Weismantel 1999). Letchford \& Lodi (2001a) use modern integer programming techniques to improve 
Young's (1968) algorithm. Letchford \& Lodi (2001b) present a polynomial time algorithm for the primal separation of Chvátal comb inequalities for the traveling salesman problem. A polynomial standard separation algorithm of Chvátal comb separation routine for Chvátal combs is presented in (?).

Primal separation can be reduced to standard separation (Padberg \& Grötschel 1985). An important question is thus whether polynomial time equivalence of the two problems holds.

The solutions to combinatorial optimization problems are often subsets of a given ground set. These solutions can thus be described by their characteristic vectors $\chi \in\{0,1\}^{n}$. With a combinatorial optimization problem of this type, one can associate the $0 / 1$ polytope, which is the convex hull of the characteristic vectors of the solutions. In light of the above question, $0 / 1$ polytopes are of particular importance.

We prove the following result.

Theorem 1.1 0/1 primal separation and 0/1 optimization are polynomial time equivalent.

As a byproduct, we have that the classical problems: 0/1 optimization, 0/1 augmentation, $0 / 1$ separation and the problem $0 / 1$ primal separation are all polynomial time equivalent.

In the second part of our paper we consider primal separation for classes of valid inequalities of several combinatorial optimization problems, whose standard version is known to be polynomial. In particular we consider inequalities for matching, stable set, maximum cut, and maximum bipartite subgraph polytopes. Although we do not provide cases where the asymptotic complexities of primal and standard separation differ, we give evidence that in all these cases primal separation is conceptually simpler and easier to implement as opposed to the corresponding standard separation. For example, we show that primal separation problem for the perfect $b$-matching polytope is easier than the corresponding standard separation variant. More precisely, our primal separation algorithm does not require an explicit minimum odd cut algorithm as it is the case for the standard separation algorithm of Padberg \& Rao (1982). Together with the above equivalence, this yields an even simpler proof that maximum weight perfect matchings and other variants of maximum matching can be computed in polynomial time.

\section{Preliminaries and notation}

A rational polyhedron $P \subseteq \mathbb{R}^{n}$ is a set of points satisfying a finite system of inequalities $A x \leq b$, where $A \in \mathbb{R}^{m \times n}$ and $b \in \mathbb{R}^{m}$ have rational components. If $P$ is bounded, then $P$ is called a rational polytope. A point $x \in P$ is called an extreme point, if it is not a convex combination of two distinct points of $P$, i.e., if $x=\alpha y+\beta z$ with $\alpha, \beta>0, \alpha+\beta=1$ and $y, z \in P$ implies that $y=z=x$. A $0 / 1$ polytope is a rational polytope $P$ whose extreme points have $0 / 1$ components. In the context of a $0 / 1$ polytope $P$, the variable $n$ denotes the dimension such that $P \subseteq \mathbb{R}^{n}$ holds. For more on $0 / 1$ polytopes see, e.g., (Ziegler 2000).

An inequality $a^{T} x \leq \beta$ is valid for a polyhedron $P$, if $P \subseteq\left\{x \mid a^{T} x \leq \beta\right\}$. An inequality $a^{T} x \leq \beta$ is tight or active at a given point $\bar{x} \in P$, if it is valid for $P$ and $a^{T} \bar{x}=\beta$.

A graph $G=(V, E)$ consists of a finite set $V$ of vertices and of a set $E$ of 2-element subsets of the vertices, called edges, i.e., $E \subseteq\{\{u, v\} \mid u, v \in V, u \neq v\}$. An edge $\{u, v\}$ is also denoted by $e$. The nodes $u$ and $v$ are called its end nodes. For $U \subseteq V$, the cut $\delta(U)$ is the set of edges $e \in E$ with $|e \cap U|=1$. Let $s$ and $t$ be nodes of $G$. An $(s, t)$-cut of $G$ is a cut $\delta(U)$ with $|U \cap\{s, t\}|=1$. A graph $G=(V, E)$ is bipartite, if there exists a cut $\delta(U)$ of $G$ with $E=\delta(U)$. 
In this paper we consider families of $0 / 1$ polytopes $\mathscr{P}$. Notice that we do not specify how the members $P \in \mathscr{P}$ of the family are encoded. For example, $\mathscr{P}$ could be the family of perfect matching polytopes $P_{G}$, where $G=(V, E)$ is an undirected graph and $P_{G}$ is the convex hull of the characteristic vectors of perfect matchings of $G$. Another family $\mathscr{P}$ could be the $0 / 1$ polytopes $P_{\phi}$ associated with propositional formulas $\phi$. A polytope $P_{\phi}$ is then the convex hull of the truth assignments of $\phi$.

Since the facet and vertex complexity (Schrijver 1986, p. 121) of a $0 / 1$ polytope $P \subseteq$ $\mathbb{R}^{n}$ is polynomial in $n$, it follows from the fundamental result of Frank \& Tardos (1987) that the $0 / 1$ optimization problem for a family $\mathscr{P}$ is strongly polynomial if and only if it is polynomial. Clearly, this also holds for $0 / 1$ separation. Therefore, every statement in the following sections involving the term "polynomial time equivalent" can as well be rephrased as "strongly polynomial time equivalent".

\section{Optimization, separation, and augmentation}

We now introduce four problems which are all connected to optimizing a linear function over a $0 / 1$ polytope. We review classical equivalence and implication results concerning these problems. In this section it will become clear that the establishment of one more implication settles the equivalence of the four problems. For the rest of the paper, let $\mathscr{P}$ denote a family of $0 / 1$ polytopes and let $P \in \mathscr{P}$.

First we define the 0/1 optimization problem. It is NP-hard to find an initial 0/1 point for $P$. However, the nature of the primal algorithms considered here, requires that we provide an initial solution.

0/1 OPTIMIZATION (0/1-OPT)

Given a $0 / 1$ point $\bar{x} \in P$ and a vector $c \in \mathbb{Z}^{n}$, find a $0 / 1$ point $\widetilde{x} \in P$ which maximizes $c^{T} x$.

We now formulate a variant of the standard separation problem for $0 / 1$ polytopes. Again, we provide an initial solution as a parameter.

0/1 SEPARATION $(0 / 1-\mathrm{SEP})$

Given a $0 / 1$ point $\bar{x} \in P$ and a point $x^{*} \in \mathbb{R}^{n}$, find a valid inequality of $P$ which separates $x^{*}$ from $P$ or assert that no such inequality exists.

The equivalence of the problems 0/1-SEP and 0/1-OPT follows from the more general result of Grötschel et al. (1981), Padberg \& Rao (1981) and Karp \& Papadimitriou (1980).

Theorem 3.1 The problems $0 / 1$-SEP and 0/1-OPT are polynomial time equivalent.

Many algorithms in combinatorial optimization, such as Ford and Fulkerson's algorithm for maximum flow, Edmonds's algorithm for maximum matching or the greedy algorithm, are primal algorithms which rely on augmentation. Here one has a feasible integral point $\bar{x}$ and has to find an augmenting solution $\widetilde{x}$, i.e., one with better objective value, or assert that $\bar{x}$ is optimal.

0/1 Augmentation (0/1-AUG)

Given a $0 / 1$ point $\bar{x} \in P$ and a vector $c \in \mathbb{Z}^{n}$, find a $0 / 1$ point $\tilde{x} \in P$

with $c^{T} \widetilde{x}>c^{T} \bar{x}$ or assert that $\bar{x}$ maximizes $c^{T} x, x \in P$. 
Schulz, Weismantel \& Ziegler (1995) and Grötschel \& Lovász (1995) have shown that $0 / 1$ augmentation and 0/1 optimization are polynomial time equivalent.

Theorem 3.2 The problems 0/1-OPT and 0/1-AUG are polynomial time equivalent.

We finally describe the $0 / 1$ primal separation problem.

0/1 PRIMAL SEPARATION (0/1-PSEP)

Given a $0 / 1$ point $\bar{x} \in P$ and a point $x^{*} \in \mathbb{R}^{n}$, find a valid inequality of $P$ which is tight at $\bar{x}$ and not valid for $x^{*}$ or assert that no such inequality exists.

The implication given in the following theorem can be found as an exercise in (Padberg \& Grötschel 1985).

Theorem 3.3 If 0/1-SEP can be solved in polynomial time, then 0/1-PSEP can be solved in polynomial time.

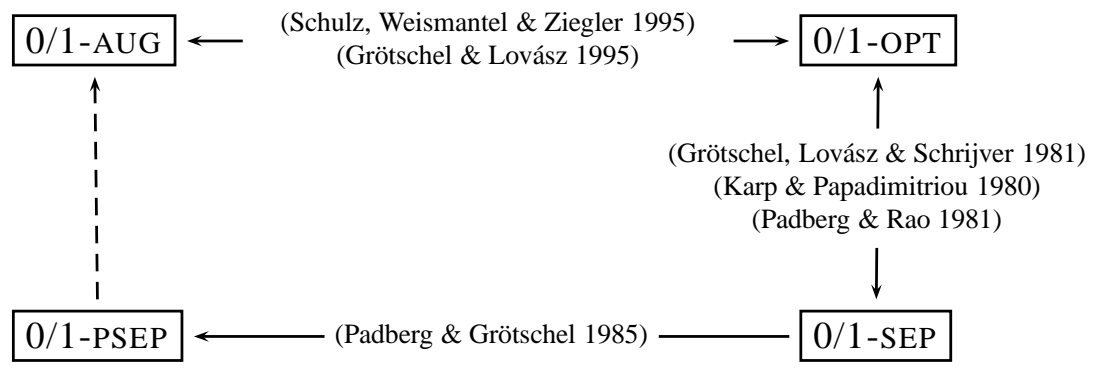

Figure 2: The implication diagram

The missing implication which settles the polynomial time equivalence of the above problems is that 0/1-PSEP implies 0/1-AUG, see Figure 2. There is an arrow from one problem A to another problem B, if B can be solved by a polynomial number of calls to an algorithm solving A and some extra polynomial time computation. The dashed implication is proved in the next section.

\section{Reducing augmentation to primal separation}

First we prove that the existence of a polynomial time algorithm for primal separation implies the existence of a polynomial time algorithm which allows us to test whether the $0 / 1$ solution $\bar{x}$ is optimal in a face of the $n$-dimensional $0 / 1$ cube intersected with $P$. Then we present an algorithm which, based on such an optimality test, constructs an augmenting solution $\widetilde{x}$.

Let $\bar{x} \in P \cap\{0,1\}^{n}$ and $J \subseteq\{1, \ldots, n\}$. The polytope $F_{J}^{\bar{x}}(P)$ is the face of $P$ obtained by fixing the variables $x_{j}, j \in J$ to the corresponding value of $\bar{x}$, i.e.,

$$
F_{J}^{\bar{x}}(P)=P \cap\left\{x \in \mathbb{R}^{n} \mid x_{j}=\bar{x}_{j}, j \in J\right\} .
$$

We consider the decision problem where one has to find out whether $\bar{x}$ is optimal in the $0 / 1$ polytope $F_{J}^{\bar{x}}(P)$ for some index set $J \subseteq\{1, \ldots, n\}$. 


TEST FACIAL OPTIMALITY (0/1-TESTOPT )
Given a $0 / 1$ point $\bar{x} \in P \cap\{0,1\}^{n}$, a vector $c \in \mathbb{Z}^{n}$ and an index set
$J \subseteq\{1, \ldots, n\}$, determine whether $\bar{x}$ maximizes $c^{T} x$ over the face
$F_{J}^{\bar{x}}(P)$.

The next lemma states that a 0/1-PSEP algorithm can be used to test facial optimality.

Lemma 4.1 If 0/1-PSEP can be solved in polynomial time, then 0/1-TESTOPT can be solved in polynomial time.

Proof: Let $T$ be the polyhedron defined by the constraints of $F_{J}^{\bar{x}}(P)$ which are tight at $\bar{x}$. By complementary slackness, $\bar{x}$ maximizes $c^{T} x$ over $F_{J}^{\bar{x}}(P)$ if and only if $\bar{x}$ optimizes $c^{T} x$ over $T$. Notice that a 0/1-PSEP algorithm for $\bar{x}$ and $P$ is a standard separation algorithm for $T$. Thus the lemma follows by the equivalence of standard separation and optimization.

Lemma 4.2 Given a 0/1-point $\bar{x} \in P$, and a vector $c \in \mathbb{Z}^{n}, 0 / 1$-AUG can be solved by $n+1$ calls to a 0/1-TESTOPT algorithm.

Proof: We iteratively construct an augmenting $0 / 1$ solution $\widetilde{x}$ of $\bar{x}$. After the $i$-th step, we will have constructed the first $i$ components $\widetilde{x}_{1}, \ldots, \widetilde{x_{i}}$ of $\widetilde{x}$ and a subset $J^{i} \subseteq\{1, \ldots, i\}$ such that the following invariant holds.

There exists an augmenting $0 / 1$ solution $\widetilde{x}$ of $\bar{x}$ contained in $F_{J i}^{\bar{x}}(P)$ and all $0 / 1$ augmenting solutions contained in $F_{J i}^{\bar{x}}(P)$ of $\bar{x}$ have first $i$ components $\widetilde{x}_{1}, \ldots, \widetilde{x}_{i}$.

We begin with a first call to a 0/1-TESTOPT algorithm to find out whether $\bar{x}$ is optimal. If the answer is yes, then there exists no augmenting solution. Otherwise, the invariant is satisfied for $i=0$ and $J=\emptyset$.

After step $i$ we proceed as follows. With a call to a 0/1-TESTOPT algorithm we find out whether $\bar{x}$ is optimal in the face $F_{J^{i} \cup\{i+1\}}^{\bar{x}}(P)$. If the answer is yes, then each augmenting $0 / 1$ solution in the face $F_{J^{i}}^{\bar{x}}(P)$ has the first $i+1$ components $\widetilde{x}_{1}, \ldots, \widetilde{x}_{i}, 1-\bar{x}_{i+1}$. By setting $J^{i+1}=J^{i}$ we ensure the invariant for $i+1$.

If the answer is no, then there exists an augmenting $0 / 1$ solution in $F_{J^{i} \cup\{i+1\}}^{\bar{x}}(P)$ and each augmenting solution in $F_{J i \cup\{i+1\}}^{\bar{x}}(P)$ has first $i+1$-st components $\widetilde{x}_{1}, \ldots, \widetilde{x}_{i}, \bar{x}_{i+1}$. By setting $J^{i+1}=J^{i} \cup\{i+1\}$ the invariant also holds in this case.

The next theorem is the missing implication from Section 3.

Theorem 4.1 If 0/1-PSEP can be solved in polynomial time, then 0/1-AUG can be solved in polynomial time.

Proof: Lemma 4.2 implies that in order to solve the augmentation problem, one needs only $n+1$ queries to a $0 / 1$-TESTOPT algorithm. The 0/1-TESTOPT problem in turn can be solved in polynomial time by Lemma 4.1 .

We can now conclude that all of the problems defined in Section 3 are polynomial time equivalent.

Theorem 4.2 The problems 0/1-OPT, 0/1-SEP, 0/1-AUG, and 0/1-PSEP are polynomial time equivalent. 


\section{Primal separation for matching polytopes}

Let $G=(V, E)$ be a graph and $a, b \in \mathbb{Z}_{+}^{V}$ be two vectors indexed by the nodes of $G$. A general simple matching of $G$ is a subset $M$ of the edge set $E$ satisfying the following condition:

$$
a_{u} \leq|M \cap \delta(u)| \leq b_{u} \quad \text { for all } u \in V .
$$

If we are given a vector of edge weights $c \in \mathbb{Z}^{E}$, then the general simple matching problem is to find a general simple matching of $G$ with maximum total weight $\sum_{e \in M} c_{e}$.

There are several special versions of a general simple matching. When $a$ is the zero vector, we call $M$ a simple $b$-matching; when $a=b$ we call $M$ a perfect simple $b$-matching. In case $b$ is a vector of all 1's, we use the terminology simple matching and perfect simple matching respectively. Observe that in order to have a nonempty set of perfect ( $b$-)matchings of $G$, the sum $\sum_{u \in V} b_{u}$ must be even*.

With a general simple matching $M$ we associate a characteristic vector $\chi^{M} \in \mathbb{R}^{E}$, whose component $\chi_{e}^{M}$ is equal to 1 if $e \in M$ and 0 otherwise.

An integral vector $x \in \mathbb{R}^{E}$ satisfies the following system of inequalities

$$
\begin{aligned}
a_{u} \leq \sum_{e \in \delta(u)} x_{e} & \leq b_{u} \text { for all } u \in V \\
0 \leq x_{e} & \leq 1 \quad \text { for all } e \in E
\end{aligned}
$$

if and only if it is a characteristic vector of a general simple matching of $G$.

\subsection{Perfect matchings}

Let us first consider the perfect matching problem. In this case the system (2) becomes

$$
\begin{gathered}
\sum_{e \in \delta(u)} x_{e}=1 \quad \text { for all } u \in V \\
x \geq 0 .
\end{gathered}
$$

The characteristic vector of a perfect matching of $G$ is an extreme point of the polytope defined by (3). However, unless $G$ is bipartite, it has also non integral extreme points. Edmonds (1965b) showed that following system of inequalities

$$
\sum_{e \in \delta(U)} x_{e} \geq 1 \text { for all } U \subset V,|U| \text { odd },
$$

is satisfied by the characteristic vector of a perfect matching of $G$. Moreover, he proved that the inequalities (3)-(4) describe a $0 / 1$ polytope. This means that the perfect matching problem can be solved by maximizing a linear function over the polytope defined by (3)-(4).

Padberg \& Rao (1982) showed that, given $x^{*} \in \mathbb{R}^{E}$, the separation problem for the inequalities (4) can be solved by finding a minimum weight odd cut $\delta(U)$ of $G$ with edge weights $x^{*}$. An odd cut $\delta(U)$ of $G$ is a cut such that $|U|$ is odd. Padberg and Rao described an algorithm that computes an odd cut of minimum weight by constructing a Gomory-Hu tree of the graph $G$. Their algorithm has polynomial time complexity. Therefore, by Theorem 3.1, it follows that maximum weighted perfect matchings can be computed in polynomial time.

By Theorem 3.3 it follows that one can solve the primal separation problem using a standard separation algorithm. We now provide a primal separation algorithm which does not make use of the standard counterpart and is even conceptually simpler. Therefore we 


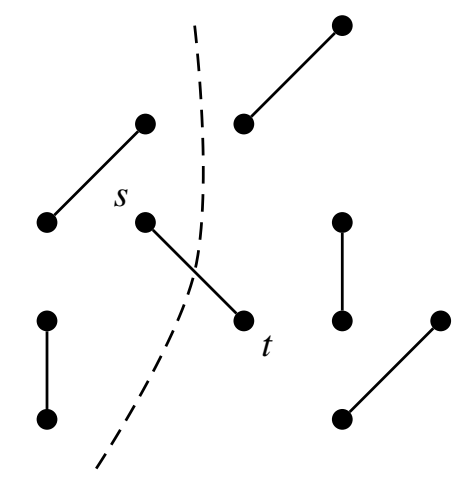

Figure 3: An odd cut triggered by $\bar{x}$. The edges drawn are those of $M$.

have a simpler proof for the polynomial time complexity of the maximum perfect matching problem than the one in (Padberg \& Rao 1982).

Let $M$ be a perfect matching of $G$ and $x^{*} \in \mathbb{R}^{E}$ be a point satisfying the inequalities (3). We want to solve the primal separation problem for the inequalities (4) with respect to the points $\bar{x}=\chi^{M}$ and $x^{*}$. To do so, we have to find a minimum weight odd cut, with respect to the edge weights given by $x^{*}$, among those that intersect $M$ in exactly one edge (see Figure 3). The minimum $x^{*}$-weight odd cut among those that contain only the edge $\{s, t\}$ of $M$ is computed by finding a minimum weight $(s, t)$-cut in the graph $G_{\{s, t\}}$ obtained from $G$ by contracting the end nodes of all edges $e \in M \backslash\{s, t\}$. These edges $e \in M \backslash\{s, t\}$ cannot belong to the odd cut we are looking for. Such a cut induces, in the obvious way, an odd cut of $G$ that intersects $M$ only in $\{s, t\}$. As the minimum weight $(s, t)$-cut can be solved with a max-flow algorithm, primal separation can thus be solved with $|V| / 2$ max-flow computations. The candidates for the edge $\{s, t\}$ are, one at the time, all the edges of the matching $M$.

Proposition 5.1 The primal separation problem for the perfect matching polytope of a graph $G=(V, E)$ can be solved with $|V| / 2$ max-flow computations.

With Theorem 1.1 this yields a very simple proof that the perfect matching problem can be solved in polynomial time. Notice that we can add edges with very small weight to $G$ to make it complete without changing the optimal solution. An initial solution $\bar{x}$ is then easily found. At this point we would like to stress that our primal separation algorithm does not require an explicit minimum odd cut algorithm. Although there exist simpler minimum weight odd cut algorithms than the original one of Padberg \& Rao (1982), see (Grötschel, Lovász \& Schrijver 1988) for an algorithm based on an inductive argument and (?) for an algorithm based on a simple implementation of Gomory-Hu cut trees, these algorithms rely on nontrivial observations, whereas the primal separation variant of the odd cut inequalities is elementary.

Corollary 5.1 The maximum weighted perfect matching problem can be solved in polynomial time.

\footnotetext{
*Sometimes in the literature, as well as in the introduction of this paper, the name "matching problem" refers to the special case when $c$ is a vector of 1's, while what we call "matching problem" is denoted by "weighted matching problem".
} 


\subsection{General matchings}

If we remove the upper bound on the variables in (2), we obtain the system

$$
\begin{array}{ll}
a_{u} \leq \sum_{e \in \delta(u)} x_{e} \leq b_{u} & \text { for all } u \in V \\
x_{e} \geq 0 & \text { for all } e \in E .
\end{array}
$$

An integral solution of this system is called a general matching of $G$, or a general perfect $b$-matching of $G$ when $a=b$. Given a vector of edge weights $c \in \mathbb{Z}^{E}$, the general matching (perfect b-matching) problem is to find a general matching (perfect $b$-matching) $x \in \mathbb{Z}^{E}$ of maximum weight $c^{T} x$.

Tutte (1954), see also (Gerards 1995), gave a polynomial time reduction from the general simple matching problem to the general perfect b-matching problem. Therefore, from now on, we will only consider the latter problem.

The system that defines a general perfect $b$-matching, i.e.,

$$
\begin{aligned}
\sum_{e \in \delta(u)} x_{e}=b_{u} & \text { for all } u \in V \\
x_{e} \geq 0 & \text { for all } e \in E,
\end{aligned}
$$

defines a polytope whose extreme points are in general not all integral.

A multiset $F$ of edges of a graph $G=(V, E)$ is a collection of the edges of $G$ containing an arbitrary number of copies of each element of $E$. The characteristic vector of a multiset $F$ is the vector $\chi^{F} \in \mathbb{R}^{E}$ where $\chi_{e}^{F}$ is the number of times that $e$ is contained in $F$. By $\delta_{F}(u)$, where $u \in V$, we denote the multiset of all the elements of $F$ that contain node $u$. A multiset of edges $B$ is called Eulerian if $\left|\delta_{B}(u)\right|$ is even for all $u \in V$.

Let $T \subseteq V$ be a subset of the nodes of $G$ with $|T|$ even. A $T$-join $J$ is a multiset of the edges of $G$ such that $\left|\delta_{J}(u)\right|$ is odd if $u \in T$ and even otherwise. A $T$-join is simple if its characteristic vector has $0 / 1$ components and is minimal if does not properly contain another $T$-join.

A perfect matching is a $V$-join of minimal cardinality, while a perfect $b$-matching is a $T$-join where $T$ is the set of nodes $u$ with $b_{u}$ odd that satisfy the additional constraints given by (6).

The $T$-join polyhedron is the set of points that can be expressed as a convex combination of the characteristic vectors of a finite set of $T$-joins. Edmonds \& Johnson (1973) showed that the $T$-join polyhedron is described by the system

$$
\begin{aligned}
\sum_{e \in \delta(U)} x_{e} & \geq 1 \text { for all } U \subseteq V,|U \cap T| \text { odd }, \\
x_{e} & \geq 0 \text { e } \in E .
\end{aligned}
$$

The general perfect b-matching polytope, is defined by (6)-(7) with $T=\left\{u \in V \mid b_{u}\right.$ odd $\}$. Generalizing a result of Edmonds (1965a), Pulleyblank (1973) showed that the general perfect $b$-matching polytope is integral.

In conclusion, we can solve the primal separation problem for the general perfect $b$ matching polytope as long as we can solve the primal separation problem for the $T$-join polyhedron.

A $T$-cut is a cut $\delta(U)$ of $G$ with $|U \cap T|$ odd. An odd cut is simply a $V$-cut. The standard separation problem of the inequalities (7) is solved by computing a minimum weight $T$-cut. An algorithm for this task was given by Padberg and Rao. This algorithm is again based on Gomory-Hu tree computation. 


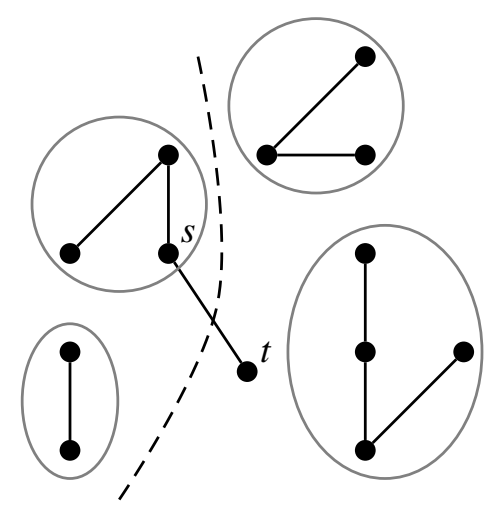

Figure 4: The connected components of $J^{\prime} \backslash\{s, t\}$ are contracted into a single node.

Let $J$ be a $T$-join of $G$ and $x^{*} \in \mathbb{R}^{E}$ be a nonnegative point. We want to solve the primal separation problem for the inequalities (7) with respect to the points $\bar{x}=\chi^{J}$ and $x^{*}$. To do so, we have to find a minimum weight $T$-cut, with respect to the edge weights given by $x^{*}$, among those that intersect $J$ in exactly one edge. The $T$-join $J$ is the union of a Eulerian multigraph $B$ and of a minimal $T$-join $J^{\prime}$. Since the intersection of each connected component of $B$ with a cut has even cardinality, we can contract all nodes of each connected component of $B$ into a single node. Recall that these components can be detected with breadth-first-search (Aho, Hopcroft \& Ullman 1974). The $T$-join $J^{\prime}$ is simple and does not contain circuits. Therefore, it is a forest, thus $\left|J^{\prime}\right|<|V|$. Furthermore, $\left|J^{\prime}\right| \leq \frac{1}{2} B$, where $B=\sum_{u \in V} b_{u}$. For each edge $\{s, t\}$ of $J^{\prime}$ we do the following. We contract all the nodes of each connected component of the forest obtained from $J^{\prime}$ by removing the edge $\{s, t\}$ (see Figure 4). Let $G^{\prime}$ be the resulting graph and let $s^{\prime}$ and $t^{\prime}$ the nodes of $G^{\prime}$ into which $s$ and $t$ have been contracted, respectively. Finally we find the minimum weight $\left(s^{\prime}, t^{\prime}\right)$-cut in $G^{\prime}$.

Such a cut induces, in the obvious way, a cut $\delta(U)$ of $G$ that intersects $J^{\prime}$ only in $\{s, t\}$. We now argue that $|U \cap T|$ is odd. This follows since

$$
\begin{aligned}
|U \cap T| & \equiv \sum_{u \in U} \delta_{J^{\prime}}(u) \quad(\bmod 2) \\
& \equiv 2\left|\left\{\{u, v\} \in J^{\prime} \mid u, v \in U\right\}\right|+1 \quad(\bmod 2)
\end{aligned}
$$

Primal separation can thus be solved with $\left|J^{\prime}\right|$ many max-flow computations.

Proposition 5.2 Let $G=(V, E)$ be a graph and $T \subseteq V$ with $|T|$ even. The primal separation problem for the $T$-join polyhedron can be reduced to $|V|-1$ maximum flow computations.

Observation 5.1 In the case of the general perfect b-matching problem, the number of nonzero entries of $\bar{x}$ is at most $\frac{1}{2} \sum_{u \in V} b_{u}$. Thus, in this case, the primal separation problem can be solved by $\min \left\{|V|-1, \frac{1}{2} \sum_{u \in V} b_{u}\right\}$ maximum flow computations.

If the reduction of Tutte (1954) is applied to a general simple matching problem, then the intersection of the solution set of the corresponding two systems (6) and (7) is a $0 / 1$ polytope. Together with the equivalence described in Section 4 the above result yields another proof that optimal general simple matchings can be computed in polynomial time. As a consequence, we obtain the following result that, in contrast to previous methods, is here obtained without explicit odd-cut algorithms. 
Corollary 5.2 The maximum weighted general simple matching problem can be solved in polynomial time.

If $a_{b}=b_{b}=2$, then one has the perfect 2-matching problem, whose associate separation problem is used to find violated 2-matching and some comb inequalities of the traveling salesman problem, see (Padberg \& Rinaldi 1990, Padberg \& Rinaldi 1991).

\section{Primal separation for stable set, cut, and bipartite subgraph polytopes}

In this section we study primal separation variants of valid inequalities for the stable set, cut, and bipartite subgraph polytopes. All variants rely, like their standard counterpart, on the computation of shortest paths. The primal separation variants here are however simpler and operate on smaller auxiliary graphs. The cut polytope, in particular, appears to fit very well into the primal separation framework since this polytope is closed under the switching operation. This means that the primal separation problem for the cut polytope can be understood as the standard separation problem for the cut cone.

\subsection{The stable set polytope}

A stable set of a graph $G=(V, E)$ is a subset $S$ of $V$ that does not contain any edge. Given a weight vector $c \in \mathbb{Z}^{V}$, the maximum weighted stable set problem is to find a stable set of $G$ of maximum weight $\sum_{u \in S} c_{u}$.

An integral vector $\bar{x} \in \mathbb{R}^{V}$ satisfies the following system of inequalities

$$
\begin{aligned}
x_{u}+x_{v} & \leq 1 \text { for all }\{u, v\} \in E, \\
x_{v} & \geq 0 \text { for all } v \in V,
\end{aligned}
$$

if and only if it is the characteristic vector $\chi^{S}$ of a stable set $S$ of $G$. The convex hull of the characteristic vectors of stable sets of $G$ is the stable set polytope of $G$. The polytope defined by (8) has integral extreme points if and only if $G$ is bipartite.

A cycle of $G$ is a minimal Eulerian set of at least three edges. A cycle is odd if it has an odd number of elements. By $V(C)$ we denote the union of the elements of a cycle $C$.

The following is the system of the odd cycle inequalities:

$$
\sum_{v \in V(C)} x_{v} \leq\left\lfloor\frac{1}{2}|V(C)|\right\rfloor \quad \text { for all odd cycles } C \text { of } G .
$$

Such a system is satisfied by the incidence vectors of all stable sets of $G$. Moreover, there are extreme points of the polytope defined by (8) that are violated by a cycle inequality.

Gerards \& Schrijver (1986) gave a polynomial time algorithm to solve the separation problem for the odd cycle inequalities. To separate a fractional point $x^{*}$ one has to find a minimal weight odd cycle of $G$, where the weight of each edge $\{u, v\}$ is given by $1-x_{u}^{*}-x_{v}^{*}$. For this one finds shortest paths in a graph obtained by making two identical copies of $G$ suitably connected. Such a graph has twice as many nodes and twice as many edges as $G$.

\subsubsection{Primal separation of odd cycle inequalities}

Here, we describe the primal separation variant of this algorithm. The advantage in this case is that one does not have to operate on a graph larger than $G$. 
Let $\bar{x}=\chi^{S}$, where $S$ is a stable set of $G$ and let $x^{*}$ satisfy (8). We want to find an odd cycle $C$, such that

$$
\sum_{v \in V(C)} x_{v}^{*}>\left\lfloor\frac{1}{2}|V(C)|\right\rfloor \quad \text { and } \sum_{v \in V(C)} \bar{x}_{v}=\left\lfloor\frac{1}{2}|V(C)|\right\rfloor .
$$

Let $Z=\left\{\{u, v\} \mid \bar{x}_{u}=\bar{x}_{v}=0\right\}$. The second condition of (10) implies that the odd cycle we want to find is one that contains one and only one edge $\{u, v\} \in Z$. Define $\bar{G}=(V, E-Z)$ and choose $\{u, v\} \in Z$. Observe that each path from $u$ to $v$ in $\bar{G}$ is an even path, since it must be an alternating sequence of nodes with value 0 and 1 with respect to $\bar{x}$. This means that, in $\bar{G}+\{u, v\}$, each cycle using the edge $\{u, v\}$ is an odd cycle. To each edge $\{u, v\}$ of $\bar{G}$ we give the weight $1-x_{u}^{*}-x_{v}^{*}$ as in the Gerards-Schrijver algorithm. Then a violated cycle can be found by computing the shortest path for such pair of nodes in $\bar{G}$ (which amounts to running $|V|$ times a shortest path algorithm) and then making a check for each edge of $Z$.

In conclusion, we have the following proposition.

Proposition 6.1 Let $G=(V, E)$ be a graph, $\bar{x}$ be a characteristic vector of a stable set of $G$ and let $x^{*} \in \mathbb{R}^{E}$ satisfy (8). The primal separation problem for odd cycle inequalities (9) with respect to $x^{*}$ and $\bar{x}$ can be solved with $|V|$ shortest path computations on a graph $\bar{G}$ which results from $G$ by deleting edges.

An undirected graph $G=(V, E)$ is $t$-perfect if the the polytope defined by (8) and (9) has integral extreme points (Chvátal 1975). Theorem 4.2 and Proposition 6.1 provide an alternative simple proof of the following assertion.

Corollary 6.1 The maximum weighted stable set problem for t-perfect graphs is solvable in polynomial time.

\subsection{The cut polytope}

Given a graph $G=(V, E)$ and a vector of edge weights $c \in \mathbb{Z}^{E}$, the maximum cut problem is to find a cut $\delta(U)$ of $G$ with maximum total weight $\sum_{e \in \delta(U)} c_{e}$. The convex hull of characteristic vectors of cuts of $G$ is the cut polytope $P_{\text {cut }}(G)$ of $G$. Equivalently, the cut polytope is the convex hull of the $0 / 1$ vectors which satisfy the following inequalities:

$$
\begin{aligned}
\sum_{e \in F} x_{e}-\sum_{e \in C-F} x_{e} & \leq|F|-1 & & \text { for all cycles } C \text { of } G \text { and } F \subseteq C,|F| \text { odd, } \\
x_{e} & \leq 1 & & \text { for all } e \in E \\
x_{e} & \geq 0 & & \text { for all } e \in E .
\end{aligned}
$$

The first set of inequalities in (11) are called the cycle inequalities. Barahona \& Mahjoub (1986) gave a polynomial time standard separation algorithm for cycle inequalities. Given $G=(V, E)$ and a fractional point $x^{*} \in \mathbb{R}^{E}$ with $0 \leq x^{*} \leq 1$ they form a new graph $G^{*}$ by duplicating the graph $G$ and by adding additional edges. The graph $G^{*}$ has twice as many nodes and four times as many edges as $G$. The standard separation problem is then solved by $|V|$ single-source shortest path computations on the graph $G^{*}$.

The maximum cut problem is one of the best suited problems for primal separation. Let $B \subseteq E$ be a subset of the edges of $G$. For a vector $x \in \mathbb{R}^{E}$, let $x^{B}$ be defined as

$$
x_{e}^{B}= \begin{cases}-x_{e} & \text { if } e \in B \\ x_{e} & \text { otherwise }\end{cases}
$$


The switching mapping is the function $r_{B}(x)=x^{B}+\chi^{B}$, where $\chi^{B}$ is the characteristic vector of $B$. The symmetric difference of two cuts is again a cut. This means that if $B$ is a cut of $G$, then the switching mapping $r_{B}(x)$ is a bijection of the characteristic vectors of cuts of $G$. In other words one has $r_{B}\left(P_{\text {cut }}(G)\right)=P_{\text {cut }}(G)$ for any cut $B=\delta(U)$ of $G$. Let $S \subseteq \mathbb{R}^{E}$ be a set of points in the space and let $B \subseteq E$. The inequality $a^{T} x \leq \beta$ is valid for $S$ if and only if $\left(a^{B}\right)^{T} x \leq \beta-a^{T} \chi^{B}$ is valid for $r_{B}(S)$.

Moreover, if $B$ is a cut of $G$ and $a^{T} x \leq \beta$ defines a facet of $P_{\text {cut }}(G)$, then also $\left(a^{B}\right)^{T} x \leq$ $\beta-a^{T} \chi^{B}$ defines a facet of $P_{\text {cut }}(G)$. As a consequence, if we know a description of the cut cone of $G$, the conical hull of all the $0 / 1$ solutions of (11), then, by switching, we can derive a description of the inequalities defining all the facets of $P_{\text {cut }}(G)$.

Let $B$ be a cut of $G$ and $x^{*} \in \mathbb{R}^{E}$. We want to solve the primal separation problem for the cut polytope with respect to the points $\bar{x}=\chi^{B}$ and $x^{*}$. Following De Simone \& Rinaldi (1994), we solve the standard separation problem for the cut cone of $G$ with respect to the point $r_{B}\left(x^{*}\right)$, producing a valid inequality $a^{T} x \leq 0$. Then the inequality $\left(a^{B}\right)^{T} x \leq-a^{T} \chi^{B}$ is violated by $x^{*}$ and is tight at $\bar{x}$.

Thus primal separation for the cut polytope is equivalent to standard separation restricted to the homogeneous inequalities or, equivalently, to standard separation for the cut cone.

\subsubsection{Primal separation for cycle inequalities}

Primal separation can also be solved with $|V|$ single source shortest paths computations. But in contrast to the known standard separation algorithm, one can again operate on the original graph. The cycle inequalities, as defined in (11), are closed under the switching operation. Therefore, primal separation amounts to restricting standard separation to homogeneous cycle inequalities, which are those whose corresponding odd set $F$ is a singleton set $F=\{f\}$. The point $x^{*}$ violates such a cycle inequality if and only if

$$
\sum_{e \in C \backslash\{f\}} x_{e}^{*}<x_{f}^{*} .
$$

This means that there exists a violated homogeneous cycle inequality if and only if there exists an edge $f=\{u, v\} \in E$ such that the length of a shortest path between $u$ and $v$ in $G$ with edge weights $x^{*}$ is strictly less than $x_{\{u, v\}}^{*}$. It is therefore enough to solve the all pairs shortest path problem for $G$ with edge weights $x^{*}$ and to compare the shortest path length between $u$ and $v$ with the value $x_{\{u, v\}}^{*}$. Remember that the all pairs shortest path problem on a graph with nonnegative edge weights can be solved with $|V|$ single-source shortest path computations, see e.g., (Ahuja, Magnanti \& Orlin 1993).

Proposition 6.2 Let $G=(V, E)$ be a graph, $\bar{x}$ be the characteristic vector of a cut of $G$ and let $x^{*} \in \mathbb{R}^{E}$ with $0 \leq x^{*} \leq 1$. The primal separation problem for the odd cycle inequalities (11) with respect to $x^{*}$ and $\bar{x}$ can be solved with $|V|$ single-source shortest path computations on $G$.

Barahona \& Mahjoub (1986) showed that the polytope defined by (11) has integral extreme points if and only if $G$ is not contractible to $K_{5}$, i.e., if it cannot be reduced to a complete graph with 5 nodes with a series contactions of the end nodes of its edges. Theorem 4.2 and Proposition 7.1 provide an alternative proof of the following assertion.

Corollary 6.2 The maximum cut problem for graphs not contractible to $K_{5}$ is solvable in polynomial time. 
There are not many other valid inequalities for the cut polytope for which standard polynomial time separation algorithms are known. Gerards (1985) gave a polynomial time standard separation algorithm for the bicycle wheel inequalities that define facets of $P_{\text {cut }}(G)$. These inequalities are not homogeneous and, unfortunately, are not closed under the switching operation. Thus Gerards' algorithm cannot be used directly for primal separation. A variation of this algorithm for the homogeneous inequalities obtained from the bicycle wheel inequalities by switching on a particular cut can be found in (Deza \& Laurent 1997, p. 481482).

\subsection{The bipartite subgraph polytope}

The maximum bipartite subgraph problem is: Given a graph $G=(V, E)$ and edge weights $c \in \mathbb{Z}^{E}$, find a subset $B \subseteq E$ such that $G_{B}=(V, B)$ is bipartite and such that $\Sigma_{e \in B} c_{e}$ is maximal. The bipartite subgraph polytope is the convex hull of characteristic vectors of such edge sets $B$. Recall that a graph is bipartite if and only if it does not contain odd cycles. The bipartite subgraph polytope of $G$ is thus the convex hull of the $0 / 1$ points satisfying the following set of inequalities

$$
\begin{aligned}
\sum_{e \in C} x_{e} & \leq|C|-1 & & \text { for all odd cycles } C, \\
x_{e} & \geq 0 & & \text { for all } e \in E, \\
x_{e} & \leq 1 & & \text { for all } e \in E .
\end{aligned}
$$

In general, the polytope defined by the system (13) has nonintegral extreme points. The graphs for which the polytope defined by the system (13) is a 0/1 polytope are called weakly bipartite. Planar graphs for example are weakly bipartite. Grötschel \& Pulleyblank (1981/82) presented a polynomial time standard separation algorithm for the inequalities (13), thus showing that the maximum bipartite subgraph problem for weakly bipartite graphs can be solved in polynomial time. Their algorithm is based on matching. To separate the inequalities (13) one can also use a specialized version of the above mentioned procedure for cycle inequalities for the max-cut problem by Barahona \& Mahjoub (1986). In this case this algorithm requires $|V|$ single-source shortest path computations on a graph $G^{*}$ with twice as many nodes and edges than $G$.

\subsubsection{Primal separation for odd cycle inequalities}

Let $\bar{x} \in\{0,1\}^{E}$ be a characteristic vector of a bipartite subgraph $G_{B}=(V, B)$ of $G$ and let $x^{*} \in \mathbb{R}^{E}$ with $0 \leq x^{*} \leq 1$. An odd cycle inequality (13) is tight at $\bar{x}$ if and only if the edges of the corresponding odd cycle $C$ that belong to $B$ form a path $P$ of length $|C|-1$. Let $f=\{u, v\}$ be the edge in $C \backslash B$. Recall that $V$ can be partitioned into two subset $U$ and $W$ which are stable in $G_{B}$. Notice now that both end nodes of $f$ are either in $U$ or $W$. The point $x^{*}$ violates the odd cycle inequality if and only if

$$
\sum_{e \in P}\left(1-x_{e}^{*}\right)<x_{f}^{*} .
$$

This means that the shortest path between $u$ and $v$ in the bipartite graph $G_{B}$ weighted with $1-x^{*}$ is less that $x_{\{u, v\}}^{*}$. We can thus solve the primal separation problem with an all pairs shortest path algorithm on $G_{B}$ with edge weights $1-x^{*}$ and by comparing, for each edge $f=\{u, v\}$ having both end nodes either in $U$ or in $W$, the distance between $u$ and $v$ in $G_{B}$ with $x_{f}^{*}$. 
Proposition 6.3 Let $G=(V, E)$ be a graph, $\bar{x} \in\{0,1\}^{E}$ the characteristic vector of a a bipartite subgraph $G_{B}=(V, B)$ of $G$ and $x^{*} \in \mathbb{R}^{E}$ with $0 \leq x^{*} \leq 1$. The primal separation problem of the odd cycle inequalities (13) with respect to $\bar{x}$ and $x^{*}$ can be solved with $|V|$ single-source shortest path computations in $G_{B}$ with edge weights $1-x^{*}$.

With Theorem 1.1 we have thus another proof of the following assertion.

Corollary 6.3 The maximum bipartite subgraph problem for weakly bipartite graphs can be solved in polynomial time.

\section{Acknowledgements}

We would like to thank the anonymous referees for their helpful remarks and suggestions.

\section{References}

Aho, A. V., Hopcroft, J. E. \& Ullman, J. D. (1974). The Design and Analysis of Computer Algorithms, Addison-Wesley, Reading.

Ahuja, R. K., Magnanti, T. L. \& Orlin, J. B. (1993). Network flows, Prentice Hall Inc., Englewood Cliffs, NJ. Theory, algorithms, and applications.

Barahona, F. \& Mahjoub, A. R. (1986). On the cut polytope, Mathematical Programming 36(2): 157-173.

Chvátal, V. (1975). On certain polytopes associated with graphs, Journal of Combinatorial Theory Ser. B 18: 138-154.

De Simone, C. \& Rinaldi, G. (1994). A cutting plane algorithm for the max-cut problem, Optimization Methods and Software 3: 195-214.

Deza, M. M. \& Laurent, M. (1997). Geometry of cuts and metrics, Springer-Verlag, Berlin.

Edmonds, J. (1965a). Maximum matching and a polyhedron with 0,1-vertices, Journal of Research of the National Bureau of Standards 69: 125-130.

Edmonds, J. (1965b). Paths, trees and flowers, Canadian Journal of Mathematics 17: 449467.

Edmonds, J. \& Johnson, E. L. (1973). Matching, Euler tours and the Chinese postman, Mathematical Programming 5: 88-124.

Firla, R. T., Spille, B. \& Weismantel, R. (1999). A primal analogue of cutting plane algorithms, Technical report, Fakultät für Mathematik, Otto-von-Guericke-Universitätt Magdeburg, Germany.

Frank, A. \& Tardos, É.. (1987). An application of simultaneous Diophantine approximation in combinatorial optimization, Combinatorica 7: 49-65.

Gerards, A. M. H. (1985). Testing the odd bicycle wheel inequalities for the bipartite subgraph polytope, Mathematics of Operations Research 10(2): 359-360. 
Gerards, A. M. H. (1995). Matching, in M. O. Ball, T. L. Magnanti, C. L. Monma \& G. L. Nemhauser (eds), Network models, Vol. 7 of Handbooks in Operations Research and Management Science, North-Holland, Amsterdam, pp. 135-224.

Gerards, A. M. H. \& Schrijver, A. (1986). Matrices with the Edmonds-Johnson property., Combinatorica 6: 365-379.

Grötschel, M. \& Lovász, L. (1995). Combinatorial optimization, in R. Graham, M. Grötschel \& L. Lovász (eds), Handbook of Combinatorics, Vol. 2, North Holland, chapter 28, pp. 1541-1597.

Grötschel, M., Lovász, L. \& Schrijver, A. (1981). The ellipsoid method and its consequences in combinatorial optimization, Combinatorica 1(2): 169-197.

Grötschel, M., Lovász, L. \& Schrijver, A. (1988). Geometric Algorithms and Combinatorial Optimization, Vol. 2 of Algorithms and Combinatorics, Springer.

Grötschel, M. \& Pulleyblank, W. R. (1981/82). Weakly bipartite graphs and the max-cut problem, Operations Research Letters 1(1): 23-27.

Gusfield, D. (1990). Very simple methods for all pairs network flow analysis, SIAM Journal on Computing 19(1): 143-155.

Karp, R. M. \& Papadimitriou, C. H. (1980). On linear characterizations of combinatorial optimization problems, 21st Annual Symposium on Foundations of Computer Science (Syracuse, N.Y., 1980), IEEE, New York, pp. 1-9.

Letchford, A. N. \& Lodi, A. (2001a). Primal cutting plane algorithms revisited, Technical report, Department of Management Science, Lancaster University.

Letchford, A. N. \& Lodi, A. (2001b). Primal separation algorithms, Technical report, Department of Management Science, Lancaster University.

Letchford, A. N. \& Lodi, A. (2002). Polynomial-time separation of simple comb inequalities, In proceedings of the 9-th Conference on Integer Programming and Combinatorial Optimization. to appear.

Padberg, M. \& Rinaldi, G. (1990). Facet identification for the symmetric traveling salesman polytope, Math. Programming 47(2, (Ser. A)): 219-257.

Padberg, M. \& Rinaldi, G. (1991). A branch-and-cut algorithm for the resolution of largescale symmetric traveling salesman problems, SIAM Review 33(1): 60 -100.

Padberg, M. W. \& Grötschel, M. (1985). Polyhedral computations, in E. L. Lawler, J. K. Lenstra, A. Rinnoy Kan \& D. B. Shmoys (eds), The Traveling Salesman Problem: A Guided Tour of Combinatorial Optimization, John Wiley \& Sons, pp. 307-360.

Padberg, M. W. \& Hong, S. (1980). On the symmetric travelling salesman problem: a computational study, Mathematical Programming Study 12: 78-107.

Padberg, M. W. \& Rao, M. R. (1981). The russian method for linear programming III: Bounded integer programming, Technical Report 81-39, New York University, Graduate School of Business and Administration. 
Padberg, M. W. \& Rao, M. R. (1982). Odd minimum cut-sets and $b$-matchings, Mathematics of Operations Research 7: 67-80.

Pulleyblank, W. R. (1973). Faces of Matching Polyhedra, PhD thesis, University of Waterloo.

Schrijver, A. (1986). Theory of Linear and Integer Programming, John Wiley.

Schulz, A. S., Weismantel, R. \& Ziegler, G. M. (1995). 0/1-integer programming: Optimization and augmentation are equivalent, ESA '95 Lecture Notes in Computer Science, number 979 in LNCS, Springer, pp. 473-483.

Tutte, W. T. (1954). A short proof of the factor theorem for finite graphs, Canadian Journal of Mathematics 6: 347-352.

Young, R. D. (1968). A simplified primal (all-integer) integer programming algorithm, $O p$ erations Res. 16: 750-782.

Ziegler, G. M. (2000). Lectures on 0/1-polytopes, Polytopes-combinatorics and computation (Oberwolfach, 1997), Birkhäuser, Basel, pp. 1-41. 